\title{
Sa1544 Risk Factors for Complications After Colonic Stent Insertion for Large Bowel Obstruction
}

Jensen T. Poon*, Steffi Yuen, Dominic Foo, Wai-Lun Law

Department of Surgery, The University of Hong Kong, Hong Kong, Hong Kong

BACKGROUND: Colonic stenting is proven to be an effective means in relieving malignant large bowel obstruction. However, severe complication such as perforation of bowel and subsequently fecal peritonitis can occur after successful of insertion of colonic stent. While colonic stenting is practiced more widely, concern also arises as a result of reports on increased complication rate from this procedure. PATIENT AND METHODS: This study analyzed the outcomes of consecutive patients who had insertion of colonic stent for large bowel obstruction in a single colorectal surgery center during November 1997 to May 2013. All patients had regular follow up in the unit after the procedure, the patient characteristics, details of the procedure, complications, subsequent outcomes and treatment were entered into a prospective database. Factors which may be associated with complications were studied. The study is approved by the Institutional Review Board of the authors' center. RESULTS: During the study period, 205 patients (129 male, mean age 68.8 year old) had colonic stent insertion. The pathology included primary colorectal cancer (151 patients), recurrent colorectal cancer (28 patients), other malignancy (23 patients) and benign stricture (3 patients). Nineteen patients had failure (reasons: persistent obstruction in 6 patients, stent migration in 12 patients, bowel perforation in 1 patients) after first stent insertion and 11 of them underwent second stent insertion which results in successful relief of obstruction in 6 patients. The overall clinical success rate of colonic stenting in relieving obstruction was $93.7 \%$. The overall complication rate after colonic stent insertion was $22.3 \%$. The incidence of each specific complication is as follows: (1) severe tenesmus (1\%; 2 patients), (2) persistent obstruction $(3.41 \% ; 7$ patients), (3) stent migration (overall 9.8\%; immediate migration after procedure in 14 patients; late migration in 6 patients but 4 of them require no further intervention), (4) recurrent obstruction due to tumor in-growth into stent $(5.4 \%$; 11 patients), (5) bowel perforation (overall in 9 patient; $4.4 \%$, immediate perforation after procedure in 2 patients; delay perforation in 7 patents). Factors including age, site of obstruction, length of stent, diameter of stent, chemotherapy were analyzed for their co-relation with complications. No particular factor was found to be associated with occurrence of persistent obstruction, recurrent obstruction stent migration but use of stent diameter size of $25 \mathrm{~mm}$ was associated with more delay bowel perforation than stent diameter size $<25 \mathrm{~mm}$ ( 6 vs. 1 perforation respectively; $\mathrm{p}=0.016$ ) CONCLUSIONS: The use of a larger diameter colonic stent $(25 \mathrm{~mm})$ does not prevent persistent obstruction, recurrent obstruction or stent migration. However, it is associated with increase chance of delayed bowel perforation. 